\title{
Stimulus-Responsive Imprinted Polymer-Based Potentiometric Sensor for Reversible Detection of Neutral Phenols
}

\author{
Longbin Qi, Rongning Liang,* and Wei Qin*
}

Cite This: Anal. Chem. 2020, 92, 4284-4291

Read Online

ACCESS | Lلll Metrics \& More | 回 Article Recommendations ｜ st Supporting Information
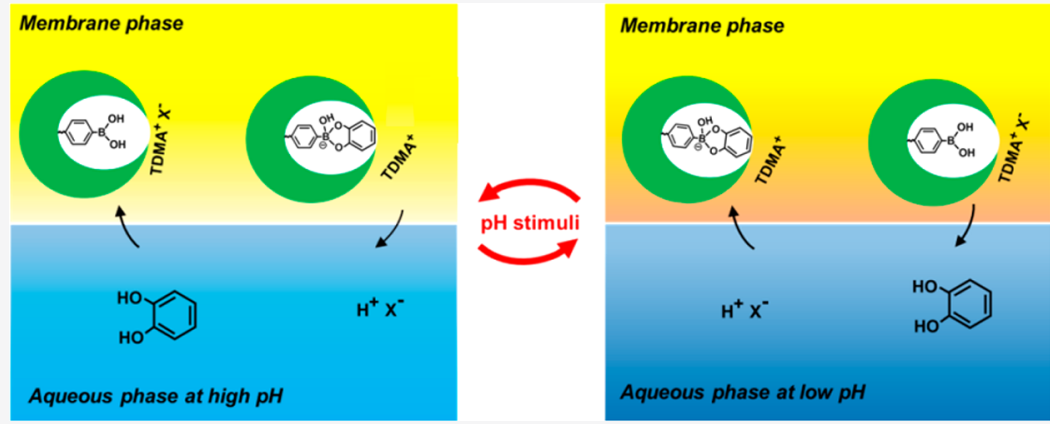

ABSTRACT: Nowadays, polymeric membrane potentiometric sensors based on the molecularly imprinted polymers (MIPs) have been successfully developed for detection of various organic and biological species. However, it is difficult for these sensors to perform reversible detection of the targets due to the high affinities of the MIPs toward the targets. In this work, we propose a novel method for fully reversible potentiometric detection of neutral phenols based on the stimulus-responsive MIP as the selective receptor. Since such smart receptor can switch its recognition abilities according to the external environmental stimuli, the MIP binding sites in the polymeric membrane can be regenerated via the stimulus after each measurement. Thus, potentiometric reversible detection of the target can be achieved. As a proof of concept, the $\mathrm{pH}$-responsive MIP is used as the selective receptor, which can be synthesized by using 4-vinylphenylboronic acid as the functional monomer. The boronate-affinity MIP can covalently bind with a cis-diol containing compound to form a five- or six-membered cyclic ester in a weakly alkaline aqueous solution, while the produced ester dissociates when the surrounding $\mathrm{pH}$ is changed to acidic. By using catechol as a model, the proposed smart sensor exhibits a significantly improved reversibility compared to the conventional MIP-based sensor. We believed that the stimulusresponsive MIP-based sensing strategy could provide an appealing way to design reversible MIP-based electrochemical and optical sensors.

$\mathrm{C}$ urrently, polymeric membrane ion-selective electrodes (ISEs) containing selective carriers (i.e., ionophores) have been widely used for detection of various ionic species in medical, environmental, and industrial analyses. ${ }^{1-3}$ Numerous selective carriers with excellent recognition capacities have been synthesized and are commercially available. ${ }^{4}$ However, most of these carriers are used for determination of inorganic ions (e.g., electrolyte ions and heavy metal ions). So far, the selective receptors used for recognitions of organic and biological species are very limited.

As highly suitable receptors for organic and biological species, molecularly imprinted polymers (MIPs) have attracted wide interest due to their excellent molecular recognition abilities toward the organic and biological targets. ${ }^{5,6}$ MIPs are less costly, easier to produce, and more stable compared to their biological counterparts such as enzymes and antibodies, and they can bind a wide range of analytes with affinities and selectivities similar to those of enzymes and antibodies. These characteristics make them particularly suitable for their use as recognition carriers of ISEs. In recent years, the MIP-based polymeric membrane ISEs have been successfully used for potentiometric detection of neutral organic molecules (e.g., chlorpyrifos, toluene $)^{7,8}$ and undissociated neutral phenols (bisphenols $\mathrm{A}, \mathrm{S}$, and $\mathrm{AF})^{9-11}$ and biological species. ${ }^{12}$ Unfortunately, despite the great success in neutral organic and biological species detection, it is difficult for these sensors to perform reversible detection of the targets because the high affinities between the receptors and their targets can result in the irreversible selective extraction process. ${ }^{1,14}$ Generally, for these MIP-based sensors, the binding targets can be removed by washing with the mixtures of water and organic solvents for

Received: October 28, 2019

Accepted: February 24, 2020

Published: February 24, 2020 


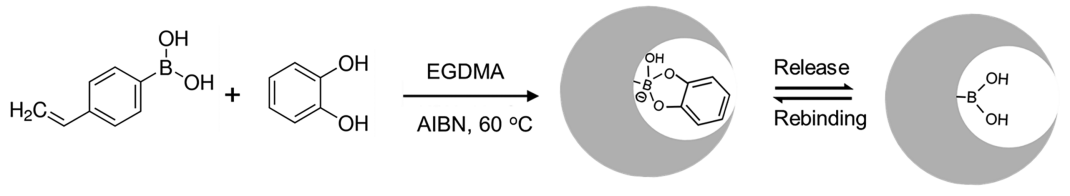

Figure 1. Schematic illustration for the synthesis of the $\mathrm{pH}$-responsive imprinted polymer.

sensor regeneration after each measurement. ${ }^{7-12}$ Nevertheless, these solvents may shorten the lifetime and reduce the response performance of the sensors since they may dissolve membrane compositions and damage membrane structures. $^{15,16}$ Therefore, it is still a big challenge for these sensors to achieve reversible detection of the targets.

Stimulus-responsive polymers, known as smart polymers, are able to respond to specific external stimuli by changing their properties such as molecular structures, surface characteristics, and dissolution behaviors. ${ }^{17,18}$ By combining the molecular imprinting technique with the stimulus-responsive materials, the stimulus-responsive MIPs have been developed, which possess both the stimulus-responsive properties and molecular recognition abilities. ${ }^{19}$ In contrast to the traditional MIPs, the recognition abilities of the stimulus-responsive MIPs can be controlled by external stimuli, such as changes in $\mathrm{pH}$, light, or temperature. The recognition of the template can be specific when the MIP maintains a 3D structure similar to the imprinting state, whereas the memory of the template will be lost when an external stimulus breaks the imprinting state. ${ }^{19}$ Inspired by the superior properties of these smart materials, nowadays a variety of stimulus-responsive MIPs have been developed including $\mathrm{pH}^{20}$ photonic, ${ }^{21}$ thermal, and dual or multiple responsive MIPs. ${ }^{22,23}$ Although these smart receptors have exhibited great success in drug delivery, biotechnology, and separation sciences, their applications in sensor development are rather rare. Up to now, there are only a few electrochemical sensors in which the stimulus-responsive MIPs were employed as the receptors. ${ }^{24,25}$ Notably, no stimulusresponsive MIP has been applied in fabricating reversible potentiometric sensors.

Herein, we propose a novel reversible potentiometric sensor based on a stimulus-responsive MIP as the receptor for detection of neutral organic species. As a proof-of-concept experiment, the boronate-affinity MIP with the $\mathrm{pH}$-responsive property is incorporated into the polymeric ISE membrane as the sensing element. Catechol, a cis-diol-containing phenol, is chosen as the template. The boronate-based MIP receptor can covalently bind with catechol to form a five-membered cyclic ester in an alkaline aqueous solution, while the produced ester dissociates when the surrounding $\mathrm{pH}$ is changed to acidic. Therefore, the regeneration of the proposed sensor can be easily achieved by changing the sample $\mathrm{pH}$. It will be shown that the stimulus-responsive MIP sensor based on covalent recognition could provide an effective way to achieve selective, sensitive, and reversible detection of neutral phenols.

\section{EXPERIMENTAL SECTION}

Reagents. 4-Vinylphenylboronic acid (VPBA), 2,2'-azobis(2-methylpropionitrile) (AIBN), ethylene glycol dimethacrylate (EGDMA), methacrylic acid (MAA), poly(vinyl chloride) (PVC), o-nitrophenyloctyl ether (o-NPOE), tridodecylmethylammonium chloride (TDMAC), and tetradodecylammonium tetrakis(4-chlorophenyl)borate (ETH 500) were purchased from Sigma-Aldrich. Catechol, sodium hydroxide, hydrochloric acid, tetrahydrofuran (THF), and sodium chloride were obtained from Sinopharm Chemical Reagent Co., Ltd. Deionized water with $18.2 \mathrm{M} \Omega \mathrm{cm}$ specific resistance was obtained by using a Pall Cascada laboratory water system. All other reagents were analytical grade and used as received.

Synthesis of the pH-Responsive MIP. The $\mathrm{pH}$ responsive MIP was synthesized by using a free radical polymerization reaction. The schematic representation for the synthesis process is shown in Figure 1. Briefly, $55 \mathrm{mg}$ of catechol and $296 \mathrm{mg}$ of VPBA were dissolved in $30 \mathrm{~mL}$ of the acetonitrile-water mixture $(1: 1, \mathrm{v} / \mathrm{v})$. The $\mathrm{pH}$ of the mixture solution was adjusted to 7.8 by using $0.1 \mathrm{M}$ sodium hydroxide. Subsequently, $2 \mathrm{~g}$ of EGDMA and $25 \mathrm{mg}$ of AIBN were added into the mixture solution. The resulting solution was purged with $\mathrm{N}_{2}$ for $30 \mathrm{~min}$ and then sealed to conduct the polymerization reaction in an oil bath at $60{ }^{\circ} \mathrm{C}$ for $24 \mathrm{~h}$. After the polymerization, the polymer was thoroughly washed with $0.1 \mathrm{M} \mathrm{HCl}$ and water successively, and then dried under vacuum at $60{ }^{\circ} \mathrm{C}$. The template removal was monitored by using an UV-vis spectrometer at the wavelength of $275 \mathrm{~nm}$. For comparison, the $\mathrm{pH}$-responsive nonimprinted polymer (NIP) was also prepared following the same procedures in the absence of the template.

Preparation of the Polymeric Membrane Electrodes. The polymeric membranes were prepared via the solvent evaporation method following our previous protocol. ${ }^{26}$ Briefly, $21.6 \mathrm{mg}$ of $\mathrm{pH}$-responsive MIP or NIP, $3.6 \mathrm{mg}$ of TDMAC, $14.4 \mathrm{mg}$ of ETH 500, $106.8 \mathrm{mg}$ of PVC, and $213.6 \mathrm{mg}$ of $o-$ NPOE were dissolved in $3.5 \mathrm{~mL}$ of THF. After vigorous stirring for $2 \mathrm{~h}$, the membrane solution was poured into a glass ring (i.d. $36 \mathrm{~mm}$ ) fixed on a glass plate. Overnight evaporation of the solvent in a chamber (temperature $25{ }^{\circ} \mathrm{C}$; humidity, $30 \%)$ yields membranes of ca. $200 \mu \mathrm{m}$. For each electrode, a disk of $5 \mathrm{~mm}$ diameter was punched from the membranes and glued to a plasticized PVC tubing with THF/PVC slurry. The inner filling solution was $0.01 \mathrm{M} \mathrm{NaCl}$. All electrodes were conditioned for $24 \mathrm{~h}$ before measurements in a solution identical to the inner filling solution.

Experimental Setup. The potentiometric measurements were performed on a $\mathrm{CHI} 760 \mathrm{C}$ electrochemical workstation under zero-current conditions in a galvanic cell: $\mathrm{Ag} / \mathrm{AgCl} / 3 \mathrm{M}$ $\mathrm{KCl} /$ sample solution/polymeric membrane/internal filling solution/3 $\mathrm{M} \mathrm{KCl} / \mathrm{AgCl} / \mathrm{Ag}$. All measurements were performed in $10 \mathrm{mM}$ phosphate buffered solution (PBS) buffer of pH 7.8 under a constant stirring at room temperature.

Electrode Regeneration. After each measurement, the $\mathrm{pH}$-responsive MIP-based electrode was treated with $10 \mathrm{mM}$ $\mathrm{HCl}$ for $10 \mathrm{~min}$ to strip catechol out of the membrane. The electrode was then reconditioned in $10 \mathrm{mM}$ PBS buffer of $\mathrm{pH}$ 7.8 for $10 \mathrm{~min}$ to restore the membrane.

Selectivity Test. The selectivity of the $\mathrm{pH}$-responsive MIPbased membrane electrode was investigated by measuring the potential responses of the proposed sensor to catechol and other analogues including dopamine, 2-aminophenol, 4-(2hydroxyethyl) phenol, and hydroquinone. To ensure that 

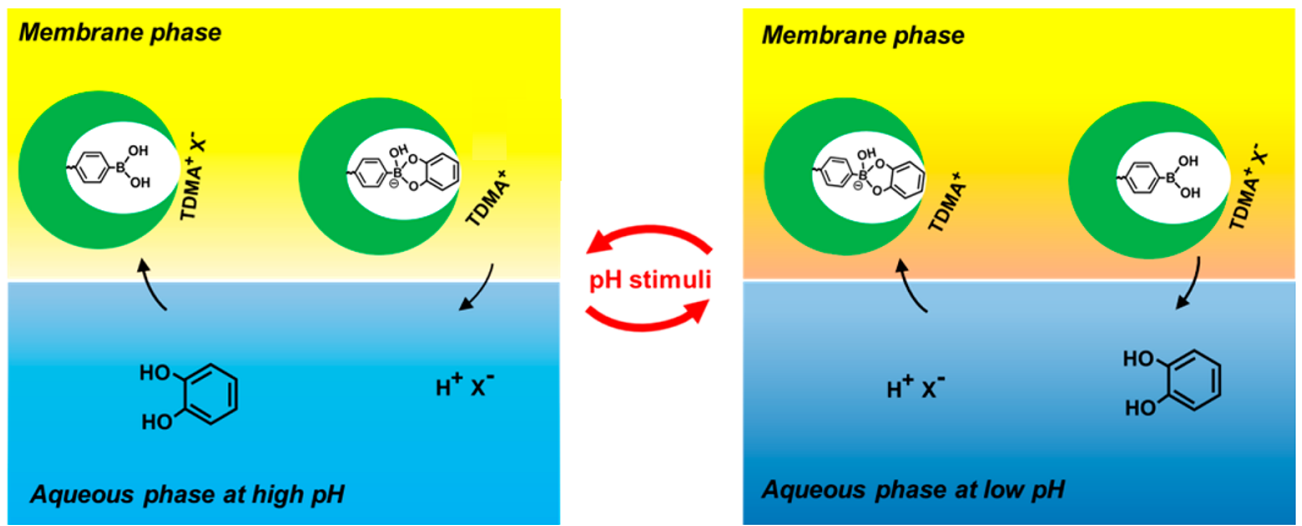

Figure 2. Possible response and regeneration mechanisms of the proposed $\mathrm{pH}$-responsive boronate-affinity MIP-based potentiometric sensor.

catechol and other phenols were in their neutral forms, a PBS buffer with a $\mathrm{pH}$ of 7.8 was selected as the background solution.

\section{RESULTS AND DISCUSSION}

Nowadays, MIP-based potentiometric sensors have been designed for detection of neutral organic species and biological species. However, to date, it is still difficult to design a reversible potentiometric sensor because of the high affinity between the MIP and the target which results in irreversible extraction of the target. In this work, we developed a novel fully reversible MIP-based potentiometric sensor for detection of neutral phenols. It is based on the boronate-affinity MIP with $\mathrm{pH}$ stimulus-responsive property as the receptor. The boronic acid groups of the MIP can covalently bind with a cisdiol containing compound to form a five- or six-membered cyclic ester in an alkaline solution while the ester dissociates when surrounding $\mathrm{pH}$ is changed to acidic. ${ }^{27}$ The $\mathrm{pH}$ dependent affinity of boronic acid group toward the target endows this MIP receptor with the $\mathrm{pH}$-responsive property. Hence, the MIP restoration in the ISE membrane can be simply accomplished by stripping the template out of the membrane with an acid solution after measurements. The new concept was evaluated for potentiometric detection of cis-diol containing phenol, catechol.

Mechanism of the pH-Responsive Sensor for Catechol Detection. The possible response and regeneration mechanisms of the proposed $\mathrm{pH}$-responsive boronate-affinity MIP-based potentiometric sensor are illustrated in Figure 2. The response principle is analogous to that of the potentiometric neutral phenol sensors described earlier. ${ }^{28}$ Under alkaline conditions, the $\mathrm{pH}$-responsive MIP exhibits high affinity toward catechol due to the formation of the fivemembered cyclic ester between the boronic acid groups of the MIP and the cis-diol group of the template. After addition of catechol into the aqueous solution, catechol can be favorably extracted from the aqueous phase into the membrane phase via the strong covalent recognition interaction between catechol and the MIP, which leads to the proton dissociation of the boronic acid group of the MIP. ${ }^{27,29}$ The dissociated proton can associate the counterion $\left(\mathrm{X}^{-}\right)$of $\mathrm{TDMA}^{+}$in the membrane and diffuse into the aqueous phase. This process facilitates the net movement of anionic species from the membrane phase to the aqueous phase and thus induces an anionic response. ${ }^{28}$

For sensor regeneration, the polymeric membrane electrode is in contact with a concentrated $\mathrm{HCl}$ solution (e.g., $10 \mathrm{mM}$ ) so that $\mathrm{HCl}$ could be coextracted into the membrane phase. ${ }^{30}$ Consequently, the $\mathrm{pH}$ of the local microenvironments around the boronate-affinity MIP may be changed. Since the MIP receptor is $\mathrm{pH}$-responsive, the above-mentioned five-membered cyclic ester can dissociate, thus releasing the catechol molecules from the MIP into the membrane. These molecules further diffuse from the membrane into the aqueous solution because of their high hydrophilicity. Subsequently, the sensing membrane is then reconditioned in PBS buffer at alkaline $\mathrm{pH}$. Thus, the boronate-affinity MIP-based polymeric ISE membrane can be completely regenerated.

Characterization of the pH-Responsive MIP. The proposed $\mathrm{pH}$-responsive MIP was synthesized by using VPBA as the functional monomer. Figure 3 shows the $\mathrm{pH}$ -

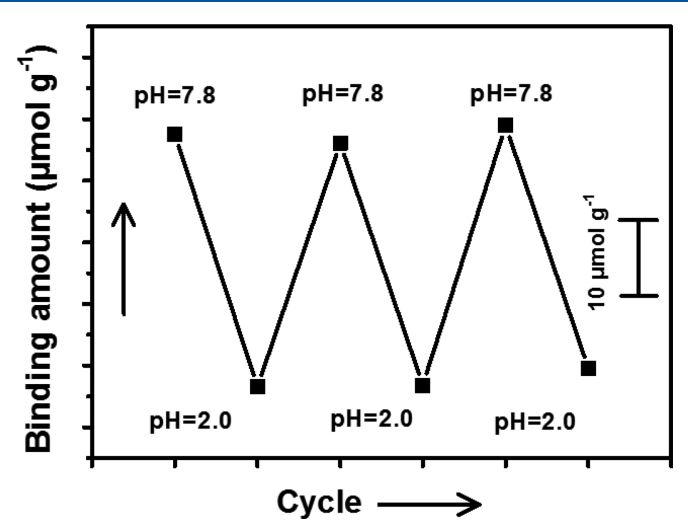

Figure 3. Changes in the catechol binding amount of the $\mathrm{pH}$ responsive MIP under alkaline (10 mM PBS buffer of $\mathrm{pH} 7.8)$ and acid conditions $(0.01 \mathrm{M} \mathrm{HCl})$. Experimental conditions: MIP, $5 \mathrm{mg} /$ $\mathrm{mL}$ MIP; catechol, $0.5 \mathrm{mM}$; incubation time, $1 \mathrm{~h}$. The detailed procedures for the binding capacity test can be found in the Supporting Information.

responsive properties of the proposed MIP. As illustrated, the boronic acid-based MIP exhibits a significant change in the binding capacity toward the template catechol at different $\mathrm{pHs}$. The binding amount of the MIP $\left(47.5 \mu \mathrm{mol} \mathrm{g}^{-1}\right)$ is much higher at $\mathrm{pH} 7.8$ than that at $\mathrm{pH} 2.0\left(9.6 \mu \mathrm{mol} \mathrm{g}^{-1}\right)$. This is due to the fact that the covalent interactions between the boronic acid and cis-diol moieties of catechol are formed at the weakly alkaline $\mathrm{pH}$ (e.g., $\mathrm{pH}$ 7.8). In this case, a high binding affinity toward the template can be observed. When the sample $\mathrm{pH}$ is switched to acidic (e.g., $\mathrm{pH} 2.0$ ), these covalent interactions can be destroyed, which results in the release of 

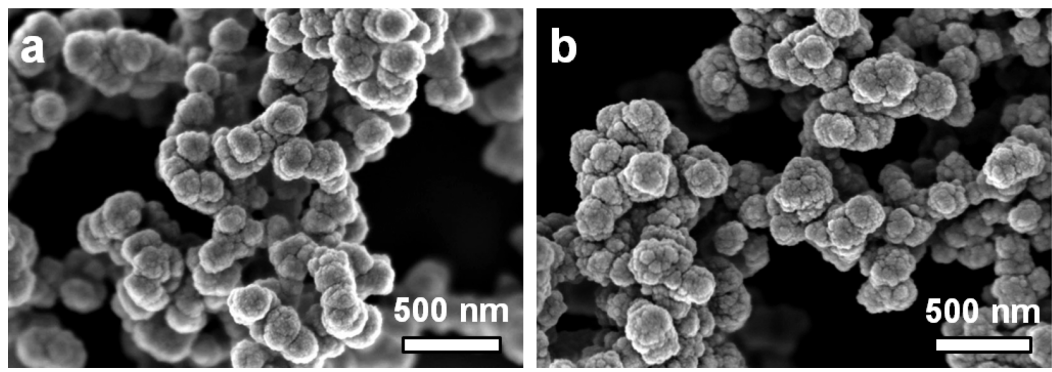

Figure 4. SEM images of the proposed $\mathrm{pH}$-responsive MIP (a) and NIP (b).

the bound templates. Figure 3 also shows that repeating the $\mathrm{pH}$-switching cycles lead to the uniformed release and uptake of catechol, which indicates the excellent reversibility of the binding configuration. These results confirm that the binding affinities of the proposed MIP are $\mathrm{pH}$-adjustable and fully reversible.

The morphologies of the $\mathrm{pH}$-responsive MIP and NIP were characterized by using the scanning electron microscopy (SEM). As shown in Figure 4a, the catechol imprinted nanoparticles are spherical with a diameter distribution of 100-200 nm. These nanoparticles can be dispersed in the polymeric ISE membrane more homogeneously than the traditional MIP microbeads, which could induce more binding sites available in the membrane and the lower membrane impedance. ${ }^{31}$ The SEM images also indicate that the NIP nanoparticles prepared with the same recipe have the similar morphological structure and particle size distribution (Figure 4b).

Potential Response of the pH-Responsive MIP-Based Membrane Electrode. The $\mathrm{pH}$-responsive MIP was used as the receptor to fabricate the electrode for selective potentiometric detection of catechol. Previous studies have revealed that electrically neutral phenols and their derivatives could generate strong anionic potential responses on quaternary ammonium salt-doped polymeric membranes under near-neutral $\mathrm{pH}$ conditions. These unexpected anionic responses can be explained by the net movement of hydrogen ions from the membrane phase to the aqueous phase stimulated by neutral phenols. ${ }^{28}$ In addition, in our previous study, it has been found that the potential responses to dissociated phenols are much lower than those to neutral phenols. ${ }^{10}$ Therefore, we explored the application of the proposed sensor in detection of neutral catechol.

The responses of the different polymeric membrane electrodes were measured to test the feasibility of using MIP as the sensing element for sensitive and selective determination of neutral catechol. As shown in Figure 5, the potential change of the boronate-affinity MIP-based electrode is much larger than those obtained by the NIP- and blank membrane-based electrodes, suggesting the specific recognition interactions between catechol and the MIP in the membrane.

Figure 6 a shows the potential responses of the proposed MIP-based sensor to catechol over a wide concentration range. It can be seen that the potential difference of the MIP-based electrode is proportional to the concentration of catechol from 1 to $80 \mu \mathrm{M}$ (Figure $6 \mathrm{~b}$ ). The potential difference between the baseline and the potential at $250 \mathrm{~s}$ after the addition of catechol was used for quantification. The detection limit of the MIP-based sensor was calculated to be $0.12 \mu \mathrm{M}(3 \sigma)$. As a comparison, the potential response of the NIP-based electrode to neutral catechol was also examined over the same

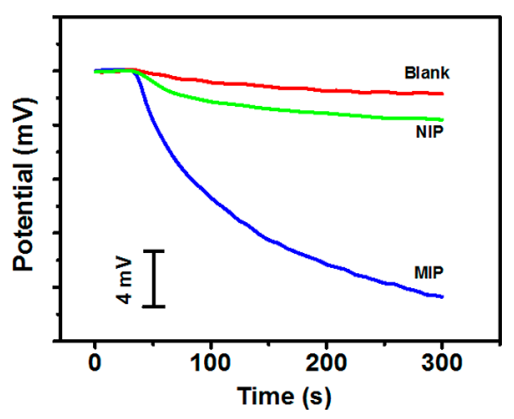

Figure 5. Potential responses of the MIP-, NIP-, and blank membrane-based electrodes to $20 \mu \mathrm{M}$ neutral catechol. Membrane compositions: MIP or NIP membrane, $6.0 \%$ MIP or NIP, $1.0 \%$ TDMAC, 4.0\% ETH 500, 29.7\% PVC, and 59.3\% $o$-NPOE; blank membrane, 1.0\% TDMAC, 4.0\% ETH 500, 31.7\% PVC, and 63.3\% oNPOE.

concentration range. Clearly, the total EMF change of the NIP-based electrode is much lower than that of the MIP-based electrode at concentrations ranging from 1 to $80 \mu \mathrm{M}$. Indeed, the calculated detection sensitivity of the NIP-based electrode $(0.21 \mathrm{mV} / \mu \mathrm{M})$ is much lower than that of the electrode based on the MIP $(0.89 \mathrm{mV} / \mu \mathrm{M})$. Such remarkable difference can be attributed to the specific recognition of the MIP for the template. ${ }^{11,26}$ These results further confirm that the measured potential changes are caused exclusively by the high-affinity binding of the imprinted polymer to the catechol molecules.

Since the potentiometric anionic response to a neutral phenol is facilitated by a decrease in the concentrations of both $\mathrm{X}^{-}$and $\mathrm{H}^{+}$in the aqueous phase, ${ }^{28}$ the effects of the concentration of $\mathrm{X}^{-}$and the sample $\mathrm{pH}$ on the potential response to catechol were investigated, respectively. The calibration curves of the $\mathrm{pH}$-responsive MIP-based electrodes at different concentrations of $\mathrm{X}^{-}$but at a fixed $\mathrm{pH}$ in the background were measured. The results are shown in Supporting Information Figure S1. As can be seen, a smaller anionic response can be observed at higher concentrations of $\mathrm{X}^{-}$, which is probably due to the fact that the concomitant ejection of HX from the membrane phase into the aqueous phase could be suppressed by the $\mathrm{X}^{-}$ions in the sample solution. Similarly, the anionic response to neutral catechol is also facilitated by the decrease in the concentration of $\mathrm{H}^{+}$ion in the aqueous phase (see Supporting Information Figure S2). Note that, when the sample $\mathrm{pH}$ is relatively far from the $\mathrm{p} K_{\mathrm{a}}$ of catechol, no obvious $\mathrm{pH}$ effect can be observed. Such similar observation has been obtained by Umezawa et al. ${ }^{28}$

Selectivity of the pH-Responsive MIP-Based Sensor. The selectivity of the proposed MIP-based potentiometric sensor was investigated by measuring the potential responses to catechol and other structure analogues including dopamine, 

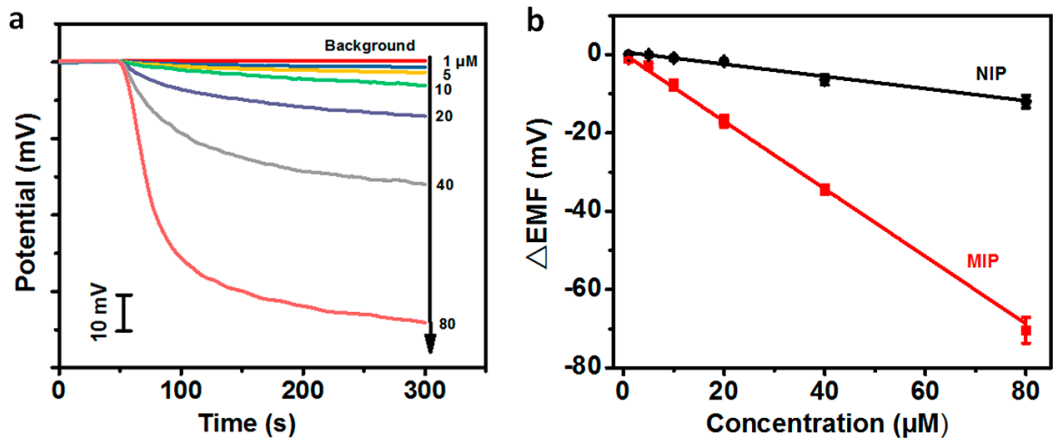

Figure 6. (a) Dynamic potential response profiles of the pH-responsive MIP-based electrode. (b) Calibration curves for the electrodes based on the pH-responsive MIP and NIP. Membrane composition: 6.0\% MIP (or NIP), 1.0\% TDMAC, 4.0\% ETH 500, 29.7\% PVC, and 59.3\% o-NPOE. The potential difference between the baseline and the potential measured at $250 \mathrm{~s}$ after the catechol addition was used for quantification. The background medium was $10 \mathrm{mM}$ PBS of $\mathrm{pH}$ 7.8. Error bar represents one standard deviation for three measurements.

2-aminophenol, 4-(2-hydroxyethyl) phenol, and hydroquinone. To ensure that catechol and other phenols are all in their neutral forms, $10 \mathrm{mM}$ PBS buffer of $\mathrm{pH} 7.8$ was used as the background medium.

As illustrated in Figure 7, the proposed sensor shows a much higher response to catechol than other analogues in terms of

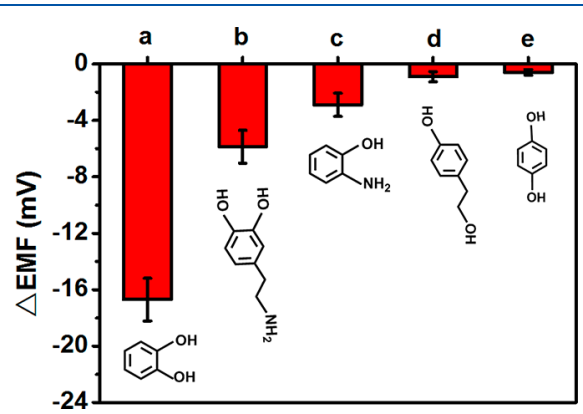

Figure 7. Potential responses of the $\mathrm{pH}$-responsive MIP-based electrode to catechol and other analogues: (a) catechol; (b) dopamine; (c) 2-aminophenol; (d) 4-(2-hydroxyethyl) phenol; (e) hydroquinone. The concentrations of catechol and other analogues were $20 \mu \mathrm{M}$ and $100 \mu \mathrm{M}$, respectively. The background medium was $10 \mathrm{mM}$ PBS of $\mathrm{pH}$ 7.8. Error bar represents one standard deviation for three measurements.

the EMF change, even if the concentration of the analogue is 5 -times higher than that of catechol. For measurement of 20 $\mu \mathrm{M}$ catechol in $10 \mathrm{mM}$ PBS, the EMF change is $16.7 \mathrm{mV}$, while those for $100 \mu \mathrm{M}$ dopamine, 2-aminophenol, 4-(2hydroxyethyl) phenol, and hydroquinone are 5.9, 2.9, 0.9, and $0.6 \mathrm{mV}$, respectively. These results demonstrate that the $\mathrm{pH}$ responsive MIP-based electrode has excellent selectivity toward catechol. Interestingly, dopamine, which also has a cis-diol group, induces a relatively larger potential change than other phenols. Indeed, the boronic acid groups of the MIP could covalently bind with the cis-diol group of dopamine, which facilitates the extraction of dopamine from the aqueous phase into the membrane phase. However, the change induced by dopamine is still much smaller than that of catechol. Hence, it can be deduced that the excellent selectivity of the proposed sensor is not only from the formation of the covalent bonds between the boronic acid groups of the MIP and the cis-diol group of the template but also from the imprinting effects, such as the $3 \mathrm{D}$ shape matching between the well-fabricated imprinting cavities and the target molecules.
Reversibility of Potentiometric Detection for Catechol. Traditionally, the MIP-based potentiometric sensors are usually regenerated by using the mixtures of water and the organic solvents. $^{7-12}$ However, the organic solvents may dissolve some membrane compositions and thus shorten the lifetime of the sensors. In this work, the $\mathrm{pH}$-responsive boronate-affinity MIP receptor was incorporated in the sensing membrane. Hence, the membrane restoration can be accomplished by simply changing the sample $\mathrm{pH}$. Reversible potentiometric detection of catechol was investigated by monitoring the potential responses of the $\mathrm{pH}$-responsive MIP-based sensor to $0.1 \mathrm{mM}$ catechol. After each measurement, a $10 \mathrm{mM} \mathrm{HCl}$ solution was employed to regenerate the electrode. As shown in Figure 8, no obvious decrease in the

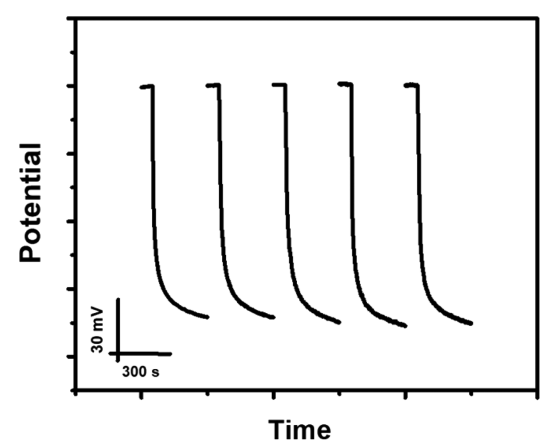

Figure 8. Regenerability of the $\mathrm{pH}$-responsive MIP-based electrode for potentiometric detection of $0.1 \mathrm{mM}$ catechol. After each measurement, the membrane electrodes were regenerated by using $10 \mathrm{mM} \mathrm{HCl}$. All electrodes were finally reconditioned in $10 \mathrm{mM}$ PBS buffer of $\mathrm{pH} 7.8$ for $10 \mathrm{~min}$ before next measurement.

potential response is observed after successively regeneration cycles for the electrode regenerated by the $10 \mathrm{mM} \mathrm{HCl}$ solution. Such results suggest that the proposed $\mathrm{pH}$-stimulus strategy is a simple but effective way to regenerate the potentiometric sensors based on the MIPs. Additionally, the influence of the regeneration solution $\mathrm{pH}$ was investigated and the data are shown in SI Figure S3. As illustrated, $1 \mathrm{mM} \mathrm{HCl}$ cannot be used to effectively regenerate the electrode, which is probably due to the weak extraction of such acid solution; on the other hand, when the concentration of the regeneration solution is above $10 \mathrm{mM}$, the present sensor can be completely regenerated. 
For comparison, the performances of the $\mathrm{pH}$-responsive MIP-based electrode regenerated by using the traditional water-organic solvent solutions were also investigated (SI Figure S4). Clearly, the potential response of the electrode regenerated by the water-ethanol solution $(3: 1, \mathrm{v} / \mathrm{v})$ to 0.1 $\mathrm{mM}$ catechol decreases obviously with increasing the regeneration cycle. After five cycles, such response decreases by $31 \%$ of its initial value (SI Figure S4a). This response behavior is very similar to that of the electrode without regeneration (SI Figure S4b). This is probably due to the fact that for each measurement, catechol can be extracted from the aqueous phase into the sensing membrane phase, while the extracted catechol molecules cannot be washed out of the membrane since the ethanol washing solution is unable to destroy the covalent bonds between catechol and the MIP. Such extraction process can gradually reduce the number of the available binding sites in the membrane, thus decreasing the subsequent potential response to catechol. Moreover, the proposed potentiometric sensor suffers a complete loss of their potential response when pure ethanol is used as the washing solution (SI Figure S4c). Further, experiments indicate that the water-acetic acid solution (3:1, v/v) of $\mathrm{pH} 2.0$ also cannot be used to effectively regenerate the electrode since a response decrease with an increased noise level can be observed (SI Figure S4d). This might be attributed to the fact that acetic acid as a solvent may dissolve some of the membrane components (e.g., plasticizer, ion-exchanger and lipophilic salt) and change the membrane properties, thus affecting the potential response to catechol. Further discussions about the effects of the organic solvents can be found in the Supporting Information. Hence, it can be demonstrated that the inorganic acid solution may be more suitable for sensor regeneration than the organic acid solution.

Potential Response Model of the Proposed Sensor for Catechol. The theoretical treatment of the response model of the present sensor is made under the following assumptions: (1) the concentration of catechol is low (normally less than $1 \mathrm{mM}$ ) and the interaction between catechol and $\mathrm{TDMA}^{+}$in the membrane is negligible; (2) for simplicity, the counterion of $\mathrm{TDMA}^{+}$in the membrane is a monovalent anion, expressed as $X^{-}$.

The sensing principle of the present sensor involves three processes. One is the extraction of catechol $(\mathrm{ROH})$ from the aqueous phase (aq) into the polymeric membrane phase $(\mathrm{m})$ with the following equilibrium:

$$
\mathrm{ROH}_{(\mathrm{aq})} \rightleftharpoons \mathrm{ROH}_{(\mathrm{m})}
$$

This equilibrium can be quantified by the partition coefficient of catechol, $K_{1}$ :

$$
K_{1}=\frac{\left[\mathrm{ROH}_{(\mathrm{m})}\right]}{\left[\mathrm{ROH}_{(a q)}\right]}
$$

The second process is the reaction between catechol and the binding sites of the boronate-affinity $\mathrm{MIP}\left(\mathrm{MIPB}_{(\mathrm{m})}\right)$ to form the five-membered cyclic ester $\left(\mathrm{MIPBR}^{-}{ }_{(\mathrm{m})}\right)$ in the membrane phase. This process can be described as

$$
\mathrm{ROH}_{(\mathrm{m})}+\mathrm{MIPB}_{(\mathrm{m})} \rightleftharpoons \mathrm{MIPBR}_{(\mathrm{m})}^{-}+\mathrm{H}_{(\mathrm{m})}^{+}
$$

with the corresponding formation constant, $K_{2}$ :

$$
K_{2}=\frac{\left[\mathrm{MIPBR}_{(m)}^{-}\right]\left[H_{(m)}^{+}\right]}{\left[\mathrm{ROH}_{(m)}\right]\left[\mathrm{MIPB}_{(m)}\right]}
$$

The third process is the diffusion of $\mathrm{HX}$ into the aqueous solution with the following equilibrium:

$$
\mathrm{H}^{+}{ }_{(\mathrm{m})}+\mathrm{X}_{(\mathrm{m})}^{-} \rightleftharpoons \mathrm{H}^{+}{ }_{(\mathrm{aq})}+\mathrm{X}^{-}{ }_{(\mathrm{aq})}
$$

This equilibrium can be quantified by the partition coefficient of $\mathrm{HX}, \mathrm{K}_{3}$ :

$$
K_{3}=\frac{\alpha_{H^{+}} \alpha_{X^{-}}}{\left[H_{(m)}^{+}{ }_{(m)}\right]\left[X_{(m)}^{-}\right]}
$$

Inserting eqs 1 and 3 into eq 2 gives

$$
K_{2}=\frac{\frac{\alpha_{H^{+} \alpha_{X^{-}}}}{K 3\left[X_{(m)}^{-}\right]}\left[\operatorname{MIPBR}_{(m)}^{-}\right]}{K_{1}\left[\mathrm{ROH}_{(\mathrm{aq})}\right]\left[\operatorname{MIPB}_{(m)}\right]}
$$

According to the mass balance in the membrane phase, $\left[\mathrm{MIPB}_{(\mathrm{m})}\right]$ can be expressed as

$$
\left[\operatorname{MIPB}_{(\mathrm{m})}\right]=\left[\operatorname{MIPB}_{(\mathrm{m})}\right]_{0}-\left[\operatorname{MIPBR}_{(\mathrm{m})}^{-}\right]
$$

where $[\mathrm{MIPB}(\mathrm{m})]_{0}$ is the initial concentration of the boronateaffinity MIP in the membrane.

According to the electroneutrality conditions in the membrane phase, the related mass values in the membrane phase can be described as

$$
\left[\operatorname{TDMA}_{(\mathrm{m})}^{+}\right]_{0}+\left[\mathrm{H}^{+}{ }_{(\mathrm{m})}\right]=\left[\mathrm{X}_{(\mathrm{m})}^{-}\right]+\left[\mathrm{MIPBR}_{(\mathrm{m})}^{-}\right]
$$

where $\left[\mathrm{TDMA}^{+}(\mathrm{m})\right]_{0}$ is the initial concentration of $\mathrm{TDMA}^{+}$in the membrane.

By inserting eqs 5 and 6 into eq $4, K_{2}$ can be correlated with $\left[X^{-}(\mathrm{m})\right]$ and $\mathrm{ROH}_{(\mathrm{aq})}$ :

$$
K_{2}=\frac{\frac{\alpha_{H^{+} \alpha_{X}}}{K_{3}\left[X_{(m)}^{-}\right]}}{K_{1}\left[\operatorname{ROH}_{(a q)}\right]\left(\frac{\left[\operatorname{MIPB}_{(m)}\right]_{0}}{\left[\operatorname{TDMA}_{(m)}^{+}\right]_{0}+\frac{\alpha_{H^{+\alpha} X^{-}}}{K_{3}\left[X^{-}(m)\right.}-\left[X^{-}(m)\right]}-1\right)}
$$

and after rearranging,

$$
\begin{aligned}
& K_{1} K_{2} K_{3}^{2}\left[\mathrm{ROH}_{(a q)}\right]\left[X^{-}{ }_{(m)}\right]^{3}+\left(K_{1} K_{2} K_{3}^{2}\left[\mathrm{MIPB}_{(m)}\right]_{0}\left[\mathrm{ROH}_{(\mathrm{aq})}\right]-K_{1} K_{2} K_{3}{ }^{2}\left[\mathrm{TDMA}_{(m)}^{+}\right]_{0}\left[\mathrm{ROH}_{(\mathrm{aq})}\right]+K_{1} \alpha_{H}{ }^{+} \alpha_{X}{ }^{-}\right)\left[X^{-}{ }_{(m)}\right]^{2}-\left(K_{3} \alpha_{H}{ }^{+} \alpha_{X}{ }^{-}\left[\mathrm{TDMA}^{+}{ }_{(m)}\right]_{0}\right. \\
& \left.+K_{1} K_{2} K_{3} \alpha_{H}{ }^{+} \alpha_{X}{ }^{-}\left[\mathrm{ROH}_{(\mathrm{aq})}\right]\right)\left[X^{-}{ }_{(m)}\right]-\left(\alpha_{H}{ }^{+} \alpha_{X}{ }^{-}\right)^{2}=0
\end{aligned}
$$

In eq 8 , all other parameters can be treated as constants except for $\left[X^{-}(\mathrm{m})\right]$ and $\left[\mathrm{ROH}_{(\mathrm{aq})}\right]$. Hence, by solving eq 8 , $\left[X^{-}(\mathrm{m})\right]$ can be correlated with $\left[\mathrm{ROH}_{(\mathrm{aq})}\right]$. According to the
Cardano formula (see the details in the Supporting Information), the solution of $\left[\mathrm{X}_{(\mathrm{m})}^{-}\right]$for this third order equation can be obtained as 


$$
\left[X^{-}{ }_{(m)}\right]=\frac{A-B}{3}+\frac{2(B-A)}{3} \cos \frac{1}{3} \arccos \frac{1}{2} \sqrt{\frac{K_{1} K_{2} C}{K_{3}}} \frac{9 \alpha_{H^{+}} \alpha_{X^{-}}\left(3 \alpha_{H^{+}} \alpha_{X^{-}}-B A+A^{2}\right)-2 K_{1} K_{2} K_{3}^{2} C(B-A)^{3}}{\left.\sqrt{\left(3 \alpha_{H^{+}} \alpha_{X^{-}}+K_{1} K_{2} K_{3} C(B-A)^{2}\right.}\right)^{3}}
$$

Where $A, B$, and $C$ denote $\left[\operatorname{TDMA}_{(\mathrm{m})}^{+}\right]_{0},\left[\mathrm{MIPB}_{(\mathrm{m})}\right]_{0}$, and $\left[\mathrm{ROH}_{(\mathrm{aq})}\right]$, respectively.

Before the addition of catechol into the aqueous solution containing $X^{-}$, the phase boundary potential $\left(E_{1}\right)$ of the polymeric membrane electrode toward $X^{-}$can be expressed as

$$
E_{1}=E^{0}-\frac{R T}{F} \ln \frac{\alpha_{X^{-}}}{\left[X_{(m)}^{-}\right]_{0}}
$$

where all constant potential contributions are included in $E^{0}$, and $R, T$, and $F$ have their usual meanings. According to the electroneutrality conditions in the membrane phase, the related mass values in the membrane phase can be described as

$$
\left[\mathrm{X}_{(\mathrm{m})}^{-}\right]_{0}=\left[\mathrm{TDMA}_{(\mathrm{m})}^{+}\right]_{0}
$$

After the addition of catechol into the aqueous solution with $X^{-}$, the phase boundary potential $\left(E_{2}\right)$ of the polymeric membrane electrode toward $X^{-}$can be described as

$$
E_{2}=E^{0}-\frac{R T}{F} \ln \frac{\alpha_{X^{-}}}{\left[X^{-}{ }_{(m)}\right]}
$$

The potential response to catechol in the sample solution can be expressed as the difference between $E_{1}$ and $E_{2}$ :

$$
\Delta E=E_{2}-E_{1}
$$

Combination of eqs 9, 10, 11, and 12 with eq 13 yields

$$
\Delta E=\frac{R T}{F} \ln \frac{\frac{A-B}{3}+\frac{2(B-A)}{3} \cos \frac{1}{3} \arccos \frac{1}{2} \sqrt{\frac{K_{1} K_{2} C}{K_{3}} \frac{9 \alpha_{H^{+}}+\alpha_{X}-\left(3 \alpha_{H^{+}}+\alpha_{X}--B A+A^{2}\right)-2 K_{1} K_{2} K_{3}^{2} C(B-A)^{3}}{\left.\sqrt{\left(3 \alpha_{H}+\alpha_{X} X^{-}+K_{1} K_{2} K_{3} C(B-A)^{2}\right.}\right)^{3}}}}{A}
$$

where $A, B$, and $C$ denote $\left[\operatorname{TDMA}_{(\mathrm{m})}^{+}\right]_{0},\left[\mathrm{MIPB}_{(\mathrm{m})}\right]_{0}$, and $\left[\mathrm{ROH}_{(\mathrm{aq})}\right]$, respectively.

eq 14 indicates that the main factors influencing the potential response of the MIP-based polymeric membrane electrode to a neutral phenol include the concentrations of the phenol and coexisting anion in the sample solution, the sample $\mathrm{pH}$, and the concentration of the anion exchanger and the MIP binding sites in the membrane. By controlling other parameters, the potential measured can be directly related to the concentration of the phenol in the sample.

\section{CONCLUSIONS}

In this work, a smart potentiometric sensor for fully reversible detection of neutral phenol is proposed for the first time. The proposed sensor is based on the boronate-affinity MIP with the $\mathrm{pH}$-responsive property as the smart receptor. The regeneration of the proposed smart sensor can be easily achieved by using a hydrochloric acid solution. The proposed sensor based on covalent recognition could provide an effective way to achieve selective, sensitive, and reversible detection of neutral phenols. Since the proposed sensing system has the flexibility of incorporating different MIPs with various stimulusresponsive properties, such as $\mathrm{pH}$, photonic, and thermal responsive properties, it can be envisioned that the novel concept is promising for fabrication of various smart electrochemical or optical membrane sensors for sensitive, selective, and reversible detection of a wide range of targets.

\section{ASSOCIATED CONTENT}

\section{SI Supporting Information}

The Supporting Information is available free of charge at https://pubs.acs.org/doi/10.1021/acs.analchem.9b04911.

Experimental details about the binding capacity test, calculations, and influences of the concentration and $\mathrm{pH}$ of the background solution as well as the effects of the type and $\mathrm{pH}$ of the regeneration solution (PDF)

\section{AUTHOR INFORMATION}

\section{Corresponding Authors}

Rongning Liang - CAS Key Laboratory of Coastal Environmental Processes and Ecological Remediation, Yantai Institute of Coastal Zone Research (YIC), Chinese Academy of Sciences (CAS); Shandong Key Laboratory of Coastal Environmental Processes, YICCAS, Yantai, Shandong 264003, P. R. China; Center for Ocean Mega-Science, Chinese Academy of Sciences, Qingdao, Shandong 266071, P. R. China; (i) orcid.org/0000-0002-9693-1242; Email: rnliang@ yic.ac.cn; Fax: +86-535-2109000

Wei Qin - CAS Key Laboratory of Coastal Environmental Processes and Ecological Remediation, Yantai Institute of Coastal Zone Research (YIC), Chinese Academy of Sciences (CAS); Shandong Key Laboratory of Coastal Environmental Processes, YICCAS, Yantai, Shandong 264003, P. R. China; Laboratory for Marine Biology and Biotechnology, Pilot National Laboratory for Marine Science and Technology (Qingdao), Shandong 266237, P. R. China; Center for Ocean Mega-Science, Chinese Academy of Sciences, Qingdao, Shandong

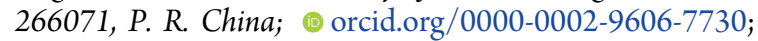
Email: wqin@yic.ac.cn

\section{Author}

Longbin Qi - CAS Key Laboratory of Coastal Environmental Processes and Ecological Remediation, Yantai Institute of Coastal Zone Research (YIC), Chinese Academy of Sciences (CAS); Shandong Key Laboratory of Coastal Environmental Processes, YICCAS, Yantai, Shandong 264003, P. R. China; University of Chinese Academy of Sciences, Beijing 100049, P. R. China

Complete contact information is available at:

https://pubs.acs.org/10.1021/acs.analchem.9b04911

\section{Notes}

The authors declare no competing financial interest. 


\section{ACKNOWLEDGMENTS}

This work was financially supported by the National Natural Science Foundation of China (21874151, 21677172, 41576106), the National Key Research and Development Program of China (2016YFC1400700), the Youth Innovation Promotion Association of CAS (2014190), and the Taishan Scholar Program of Shandong Province (tspd20181215).

\section{REFERENCES}

(1) Zdrachek, E.; Bakker, E. Anal. Chem. 2019, 91, 2-26.

(2) Bobacka, J.; Ivaska, A.; Lewenstam, A. Chem. Rev. 2008, 108, 329-351.

(3) Pankratova, N.; Cuartero, M.; Cherubini, T.; Crespo, G. A.; Bakker, E. Anal. Chem. 2017, 89, 571-575.

(4) Bühlmann, P.; Pretsch, E.; Bakker, E. Chem. Rev. 1998, 98, $1593-1688$.

(5) Haupt, K.; Mosbach, K. Chem. Rev. 2000, 100, 2495-2504.

(6) Wulff, G. Chem. Rev. 2002, 102, 1-28.

(7) Liang, R.; Song, D.; Zhang, R.; Qin, W. Angew. Chem., Int. Ed. 2010, 49, 2556-2559.

(8) Liang, R.; Chen, L.; Qin, W. Sci. Rep. 2015, 5, 12462.

(9) Kamel, A. H.; Jiang, X.; Li, P.; Liang, R. Anal. Methods 2018, 10, 3890-3895.

(10) Wang, T.; Liang, R.; Yin, T.; Yao, R.; Qin, W. RSC Adv. 2016, 6, 73308-73312.

(11) Zhang, H.; Yao, R.; Wang, N.; Liang, R.; Qin, W. Anal. Chem. 2018, 90, 657-662.

(12) Liang, R.; Ding, J.; Gao, S.; Qin, W. Angew. Chem., Int. Ed. 2017, 56, 6833-6837.

(13) Shvarev, A.; Bakker, E. Anal. Chem. 2005, 77, 5221-5228.

(14) Shvarev, A.; Bakker, E. J. Am. Chem. Soc. 2003, 125, 1119211193.

(15) Papp, S.; Jágerszki, G.; Gyurcsányi, R. E. Angew. Chem., Int. Ed. 2018, 57, 4752-4755.

(16) Nakamura, T.; Rechnitz, G. A. Anal. Chem. 1985, 57, 393-394.

(17) De las Heras Alarcón, C.; Pennadam, S.; Alexander, C. Chem. Soc. Rev. 2005, 34, 276-285.

(18) Islam, M. R.; Lu, Z.; Li, X.; Sarker, A. K.; Hu, L.; Choi, P.; Li, X.; Hakobyan, N.; Serpe, M. J. Anal. Chim. Acta 2013, 789, 17-32.

(19) Xu, S.; Lu, H.; Zheng, X.; Chen, L. J. Mater. Chem. C 2013, 1, $4406-4422$.

(20) Li, L.; Lu, Y.; Bie, Z.; Chen, H.-Y.; Liu, Z. Angew. Chem., Int. Ed. 2013, 52, 7451-7454.

(21) Renkecz, T.; Mistlberger, G.; Pawlak, M.; Horváth, V.; Bakker, E. ACS Appl. Mater. Interfaces 2013, 5, 8537-8545.

(22) Xu, L.; Pan, J.; Dai, J.; Li, X.; Hang, H.; Cao, Z.; Yan, Y. J. Hazard. Mater. 2012, 233-234, 48-56.

(23) Ma, Y.; Zhang, Y.; Zhao, M.; Guo, X.; Zhang, H. Chem. Commun. 2012, 48, 6217-6219.

(24) Wei, Y.; Zeng, Q.; Bai, S.; Wang, M.; Wang, L. ACS Appl. Mater. Interfaces 2017, 9, 44114-44123.

(25) Li, Y.; Hong, M.; Bin, Q.; Lin, Z.; Cai, Z.; Chen, G. J. Mater. Chem. B 2013, 1, 1044-1051.

(26) Kou, L.; Liang, R.; Wang, X.; Chen, Y.; Qin, W. Anal. Bioanal. Chem. 2013, 405, 4931-4936.

(27) Chen, Y.; Huang, A.; Zhang, Y.; Bie, Z. Anal. Chim. Acta 2019, 1076, 1-17.

(28) Ito, T.; Radecka, H.; Tohda, K.; Odashima, K.; Umezawa, Y. J. Am. Chem. Soc. 1998, 120, 3049-3059.

(29) Strawbridge, S. M.; Green, S. J.; Tucker, J. H. Chem. Commun. 2000, 2393-2394.

(30) Xie, X.; Mistlberger, G.; Bakker, E. J. Am. Chem. Soc. 2012, 134, 16929-16932.

(31) Liang, R.; Kou, L.; Chen, Z.; Qin, W. Sens. Actuators, B 2013, 188, 972-977. 